\title{
The characteristics of epilepsy in a largely untreated population in rural Ecuador
}

\author{
M Placencia, J W A S Sander, M Roman, A Madera, F Crespo, S Cascante, S D Shorvon
}

\begin{abstract}
A house-to-house survey of epileptic seizures covering a population of 72121 persons was carried out in a rural area of northern Ecuador. A cascade system of diagnosis was used to identify all cases of epileptic seizures in this population. 1029 cases were found, of whom 881 were considered to be definite cases and 148 were possible cases. Of the 1029 cases, $56 \%$ had active epilepsy. The lifetime prevalence rate was found to lie between $12 \cdot 2$ and $19 \cdot 5 / 1000$ and the prevalence of active cases between $6 \cdot 7$ and $8 \cdot 0 / 1000$. An annual incidence rate of between 122 I 100000 and 190/100 000 was estimated. Seizure type was classified without EEG data and almost half of the cases had partial seizures. In $27 \%$ of cases an aetiology was proposed on clinical grounds. This is one of few reported studies of a population that has been largely unexposed to antiepileptic drugs, providing an opportunity to study the natural history of the untreated condition. It has been suggested that treatment in newly developing epilepsy will prevent its development to a chronic condition. Only $37 \%$ of the 1029 cases had ever received antiepileptic drugs, and only $12 \%$ of the cases were taking them at the time of the survey. Despite this, a high rate of inactivity was observed, with $44 \%$ of all cases free of seizures. Nearly two thirds of the inactive cases identified had never received treatment with antiepileptic drugs. In a subgroup of untreated cases with an active condition, treatment with antiepileptic drugs was initiated and was highly effective even in cases with a long previous history. Thus the findings from this study suggest that the development of epilepsy resistant to therapy is not always associated with a long duration of untreated epilepsy.
\end{abstract}

(F Neurol Neurosurg Psychiatry 1994;57:320-325)

The study of the natural history of epilepsy in a general population is important for an understanding of the condition, an assessment of the effectiveness of treatment, and the planning of provision of health care. Most studies of the characteristics of epilepsy have been carried out from a hospital base. This introduces a selection bias towards chronic and severe cases, a problem particularly for studies of natural history. ${ }^{1}$ Only a few persons with epilepsy or a history of epileptic seizures attend hospital regularly, and it is self-evident that the natural history of the condition cannot be easily studied. There have been a few community or population based studies, in which the occurrence of epilepsy has been studied in detail, but these have seldom been comprehensive. ${ }^{23}$. The methodology of published studies has been criticised, especially in regard to identification and classification of cases. ${ }^{4}$ Furthermore, the most comprehensive studies have been from the developed world, in which most patients have sought medical attention, and have received treatment. It is a remarkable fact that the natural history of untreated epilepsy is almost unknown; effective treatment has been available for many years, bromides being introduced into clinical practice in 1857, phenobarbitone in 1912, and phenytoin in 1938. All these were introduced long before any population study of epilepsy was made. ${ }^{1}$

There have been few opportunities to study an untreated population. This is important as it has been suggested that the patterns of epilepsy in the long term might be influenced by early treatment. ${ }^{5-7}$ In treated populations, a high rate of early remission is found, with the disease lasting a short time only. It is proposed that this pattern of early remission may be due to treatment, and thus treatment may prevent the evolution of the condition to a chronic disease. Alternatively, this pattern is simply a reflection of the natural history of the condition.

The only comprehensive method to study a disease is to screen the whole population and to examine all possible cases of the condition in detail. ${ }^{4-10}$ Such a staged method does not rely on previous diagnosis or medical records, thus eliminating a major source of selection bias. A few such screening studies have been carried out for epilepsy, but often with methodology that selects cases of tonic clonic or convulsive seizures preferentially. ${ }^{11-13}$ This selectivity may be responsible for the variation in prevalence and the finding of high proportions of convulsive seizures in many studies. ${ }^{4}$ Another deficiency in published studies has been a failure to define the demographic features of the study population, which is necessary to place the findings in context for comparative purposes.

In this paper, we report the results of a comprehensive population study of the characteristics and natural history of epilepsy in a population of 72121 persons in a rural area 
of highland Ecuador. Sophisticated identification methods were used and a detailed study of epileptic seizures made. A special feature of the population, is that most patients had never received antiepileptic drug treatment, and this has presented us with an opportunity to study epilepsy in its untreated state; an opportunity that has seldom been exploited. This programme of investigation also included sociological and psychological studies, and the evaluation of a prospective treatment programme. ${ }^{14}$ These aspects are discussed elsewhere, and in this paper only the demographic and basic prognostic aspects are considered.

Two of the aims of the study were to describe the rates of non-febrile seizures (prevalence and incidence) and to describe in detail the occurrence of epileptic seizures in a defined population resident in an area of El Carchi and Imbabura province in Ecuador. Full aims and methodology are given elsewhere. ${ }^{14}$ In this paper, the terms epilepsy and epileptic seizures are used interchangeably.

\section{Methods}

STUDY DESIGN

The El Carchi and Imbabura region of Ecuador is a rural area in the high Andean region of northern Ecuador. It has an agriculturally based peasant population of largely Mestizo (mixed Amerindian and European) origins. A detailed description of the population and study area is given elsewhere..$^{1415}$

All medical graduates from Ecuadorian medical schools are obliged to spend their first year after qualification drafted to medical centres in rural areas, and are known as rural doctors ("medico rural"). The government rural medical service in Ecuador is based on primary health care, provided by a network of these medical centres spread through the country. Most of the health care centres are staffed by a rural doctor, a nursing auxiliary, and a health worker. Patients have free access to these centres, where most illnesses are dealt with. Facilities are rudimentary at this level, but doctors at the centre can refer cases to central hospitals. The specialists are concentrated in large cities and there is no neurologist in $\mathrm{El}$ Carchi or Imbabura province.

The study method is outlined here, but a more detailed description is given elsewhere. ${ }^{1416}$ The study area was comprehensively mapped with a variety of cartological methods. Prestudy preparations included information days and local publicity, which were an important way of gaining the full cooperation of the local community. All personnel involved were trained to achieve a high degree of uniformity, usually in the field and activities included conferences, prepilot trials, audiovisuals, case reviews, and meetings. A complex system of planning and monitoring was used during the survey to ensure complete coverage. Considerable effort and planning were made to ensure as complete a coverage of the population as possible as this was seen as an essential feature of the study.

Stage 1: screening questionnaire and piloting

The screening questionnaire used was given a pilot trial before the study. ${ }^{14}{ }^{16}$ It evolved from preliminary versions tested in a neurological clinic in patients with known epilepsy, other neurological conditions, and healthy controls. The sensitivity of the questionnaire in the pilot survey was $98 \%$ and its specificity $92 \%$. Details of the design, piloting, and sensitivity and specificity testing of the final nine question instrument used in the survey are given elsewhere. ${ }^{16}$

\section{Stage 2: house to house survey}

A door-to-door survey was carried out with the screening questionnaire to identify all residents with suspected or with a history of epileptic seizures. A total of 15195 dwellings were surveyed and 72121 people $(96.2 \%$ of the total estimated population for the area) were screened; of these $6330(9 \%)$ were positive.

Stage 3: examination by a rural doctor Of the 6330 positive (97\%), 6155 were then examined by one of 11 rural doctors. Standardised protocols were used, ${ }^{14}$ and on the basis of these, 1235 persons were considered positive.

\section{Stage 4: neurological examination}

A team of 12 neurologists examined 1199 stage 3 positive cases. They carried out a detailed history and examination with the same standard protocols and definitions used by the rural doctor, and diagnosed 1029 cases of afebrile seizures.

\section{Quality control}

To ascertain false negative rates at screening (defined as people who although having a history of epileptic seizures, responded negatively to the screening), a random sample of 904 people $(1 \cdot 37 \%)$ of the 65791 negatives were interviewed by a specially trained rural doctor. Of these four $(0.44 \%)$ were found to be positive, and these cases were confirmed by a neurologist. If this proportion is projected to the total number of negatives (65 791), a total of 291 false negatives (further cases) would be expected.

Two hundred and twenty seven (4.61\%) people out of the 4920 deemed as false positives (defined as those without epilepsy who responded positively to screening) by the rural doctors were examined by the neurologists. Four ( $1.76 \%)$ were found to have a history of epileptic seizures, and if this proportion is projected to the total (4930), a further 87 cases should be expected.

\section{Case review}

A stringent review of case records of the 1029 cases was also carried out by an international panel of neurologists experienced in the problems of epilepsy; 881 ( $86 \%)$, for whom data were comprehensive, were considered to 
be certain (definite) cases, and 148 were probable cases.

\section{Definitions}

The medical history was provided by the patient, or in the case of children by relatives. Almost invariably, when the medical examination was carried out, the patient was accompanied by relatives, and in almost all cases the history was extended and corroborated by family members.

For the purposes of this study, epileptic seizures were defined as the occurrence of attacks accepted as epileptic after examination by an experienced neurologist and after a review by the principal investigator and the international neurological panel. Febrile seizures were excluded, and were defined as seizures occurring between the ages of 2 and 5 years, with a definite history of fever where this was available. All other epileptic seizures were included whatever their cause or precipitant. Classification of seizure type was made according to strict criteria. This classification was based on the clinical history and on a protocol flowchart of symptoms, without EEG data, and in this sense does not conform exactly to the 1981 International League Against Epilepsy (ILAE) classification. ${ }^{17}$ The protocol flowchart used for classifying seizures is published elsewhere. ${ }^{14}$

The seizures were classified according to whether they were active or inactive. A case was considered to be active if a seizure had occurred in the previous 12 months or if the patient was taking antiepileptic drugs. A case was considered inactive if a seizure had not occurred in the previous 12 months and the patient was not taking antiepileptic drugs. The treatment state of the patient was elicited; the patient was considered to be on current treatment if antiepileptic drugs had been taken regularly for the previous four weeks or more; and to have been previously treated, if antiepileptic drugs had been taken for four weeks or more in the past; and untreated if no antiepileptic drugs had ever been taken, or had been taken irregularly for less than four weeks at a time in total.

The temporal pattern of the seizure disorder was identified. The occurrence of epileptic seizures was often carefully recorded by the patient and his family, and in the great majority of cases detailed histories were obtained. It was usually possible to categorise

Table 1 Age and sex distribution of the population screened and of all identified cases

\begin{tabular}{|c|c|c|c|c|c|c|}
\hline \multirow[b]{2}{*}{ Decades } & \multicolumn{2}{|l|}{ Males } & \multicolumn{2}{|l|}{ Females } & \multicolumn{2}{|l|}{ Total } \\
\hline & $N$ & $n$ & $N$ & $n$ & $N$ & $n$ \\
\hline $\begin{array}{c}0-9 \\
10-19 \\
20-29 \\
30-39 \\
40-49 \\
50-59 \\
60-69 \\
70-98 \\
\text { Total }\end{array}$ & $\begin{array}{r}10070 \\
9409 \\
4638 \\
3315 \\
3015 \\
2390 \\
1563 \\
1427 \\
35828\end{array}$ & $\begin{array}{r}69 \\
138 \\
60 \\
51 \\
45 \\
38 \\
17 \\
16 \\
434\end{array}$ & $\begin{array}{r}10001 \\
8856 \\
4790 \\
3842 \\
3237 \\
2568 \\
1612 \\
1388 \\
36294\end{array}$ & $\begin{array}{r}79 \\
116 \\
85 \\
90 \\
99 \\
67 \\
35 \\
24 \\
595\end{array}$ & $\begin{array}{r}20071 \\
18265 \\
9428 \\
7157 \\
6252 \\
4958 \\
3175 \\
2815 \\
72121\end{array}$ & $\begin{array}{r}148 \\
254 \\
145 \\
141 \\
144 \\
105 \\
52 \\
40 \\
1029\end{array}$ \\
\hline
\end{tabular}

$\mathrm{N}=$ sampled population; $\mathrm{n}=$ cases patients according to the total seizure numbers, into those who had had a single episode, 2-10 seizures, 11-100, and more than 100 seizures in total.

The aetiology of the seizures was based on the history and examination, without sophisticated investigations, in most cases. The presence of acute precipitants for the seizures was elicited through the history and from a checklist of possible factors.

\section{Results}

Table 1 shows the age and sex distributions of the surveyed population and of the 1029 cases with a history of seizures. The lifetime prevalence of epileptic seizures was estimated to lie between $12 \cdot 2 / 1000$ and $19 \cdot 5 / 1000$ and the prevalence of the active condition between $6 \cdot 7 / 1000$ and $8 \cdot 0 / 1000$. The lower figures represent the 881 cases considered as definite and the higher figure is an adjusted figure, calculated by the addition of a further 378 cases estimated from the various quality control steps. ${ }^{18}$ The annual incidence rates were similarly estimated to be between $122 / 100000$ and 190/1000 000. These figures are fully discussed elsewhere. ${ }^{18}$

Table 2 shows the antiepileptic drug treatment state of the 1029 cases divided into two groups; 575 (56\%) had active epilepsy and $454(44 \%)$ an inactive condition. Only 386 (37\%) had ever received treatment with antiepileptic drugs and $121(12 \%)$ were on regular treatment at the time of the survey. Seventy per cent of the patients with inactive seizures had never received treatment, or $58 \%$ of patients with active seizures. Of the 881 definite cases, only 258 (29\%) had ever received treatment with antiepileptic drugs

Table 3 shows the distribution of total seizure number in the 1029 cases. A total of $114(11 \%)$ had had a single episode, of whom 29 cases had experienced it in the 12 months before the study. In $4 \%$ of cases, seizure numbers were not reliably obtained; this often related to patients who had been in long remission. Among the 881 cases considered definite, 70 (8\%) had had a single episode, and among the 148 probable cases, $44(30 \%)$ had had a single episode.

Table 2 Treatment state for identified cases

\begin{tabular}{lllr}
\hline & Active & Inactive & Total \\
\hline On treatment at time of the survey & 121 & NA & 121 \\
Treatment only in the past & 125 & 140 & 265 \\
Ever on treatment & 246 & 140 & 386 \\
Never on treatment & 329 & 314 & 643 \\
Total & 575 & 454 & 1029 \\
\hline
\end{tabular}

NA = not applicable.

Table 3 Seizure numbers for identified cases

\begin{tabular}{lr}
\hline Single episode & 114 \\
$2-10$ seizures & 347 \\
$11-100$ seizures & 145 \\
$>100$ seizures & 382 \\
Unknown & 41 \\
Total & 1029 \\
\hline
\end{tabular}


Table 4 Seizures classification for the 881 cases estimated as definite

\begin{tabular}{lc}
\hline Seizure type & $n(\%)$ \\
\hline Partial: & $17(2)$ \\
Simple & $44(5)$ \\
Complex & $298(34)$ \\
Secondarily generalised & $78(9)$ \\
$>1$ type of partial seizure & $437(49)$ \\
Total & \\
Generalised: & $378(43)$ \\
Tonic clonic & $25(3)$ \\
Other generalised & $27(3)$ \\
$>1$ type of general seizure & $430(49)$ \\
Total & $14(2)$ \\
Unclassifiable & $881(100)$ \\
Total &
\end{tabular}

Table 4 shows the classification of seizure type among the 881 definite identified cases. There is a relatively high proportion of partial seizures, but this figure should be considered a minimum estimate, as some of the apparently generalised cases would show partial features after investigation.

No cause or acute precipitant for the seizures was identified in $73 \%$ of the 881 patients. Likely aetiologies were estimated in $27 \%$ of cases and these included birth trauma $(9 \%)$, head injury (7\%), neurocysticercosis $(3 \%)$, and cerebrovascular diseases $(3 \%)$.

\section{Discussion}

A primary purpose of this report is to provide a description of the occurrence of epilepsy in a rural population of a developing country in which most patients had never received antiepileptic drugs. The most comprehensive population based studies have been from developed countries with well organised medical systems and in such populations almost all patients will have received antiepileptic drugs at some point. ${ }^{2} 319$

There have been a number of population based studies of epilepsy in the developing world, but in these, methodology of case selection and classification have been the subject of some criticism. Commonly for instance, a screening questionnaire is employed in which epilepsy is only one of the conditions being sought and there is a bias towards the identification of tonic clonic convulsions only. ${ }^{416}$ These methodological problems are likely to distort the findings, and may explain the high proportion of generalised seizures in some studies.

The diagnosis of epilepsy-which is essentially a clinical diagnosis-is not straightforward in all cases. To overcome this problem, a staged system of case identification was used. First, we designed the questionnaire to have high sensitivity and linked this with a cascade system of examination. Thus all cases were first seen by a rural doctor, then a neurologist, and then the record was reviewed by a panel of neurologists. Secondly, each examination was carried out with standardised instruments, allowing quantification of the clinical data, and application of strict criteria, both to diagnosis and classification. Thirdly, all cases were examined and reviewed by a team of experienced neurologists who completed detailed questionnaires. This has allowed the acquisition of a detailed database of high quality, not dependent either on the surveyor or rural doctor (non-specialist). Fourthly, we accepted that there is a group of cases in whom the diagnosis is not certain and after a thorough case record review we subclassified this group as "probable cases." In assessing prevalence we prefer to give a range, rather than a single figure, to incorporate possible cases and to acknowledge this clinical problem. Furthermore, the fact that the questionnaires and the criteria used were standardised and strictly defined gives the potential for comparative data to be gathered, both within the same population and transculturally.

The lifetime prevalence of epilepsy in this population was estimated as a range $(12 \cdot 2 / 1000-20 \cdot 1 / 1000)$. The positively identified definite cases (881) form the lowest figure. The higher figure is estimated by extrapolating the false negative rates from the quality control procedures to the whole population, and adding this to the positively identified cases. The prevalence of active cases was $6 \cdot 7-8 \cdot 0 / 1000$, and this should be considered a minimum estimate of the true prevalence.

Incidence figures were derived retrospectively from the number of cases in the population whose epilepsy developed in the 12 months before the survey date. Such a retrospective method has possible drawbacks discussed elsewhere, ${ }^{4}$ but nevertheless, the incidence derived of 122-190/100 000/year is higher than in most other population studies and at least twice that expected in developed countries. ${ }^{4}$ Full discussions of the prevalence and incidence rates are published elsewhere. ${ }^{18}$

The classification of epilepsy as well as diagnosis was carried out with defined protocols and methods. ${ }^{14}$ The classification of seizure type was made from clinical features only, and not EEG (which was not available to the study workers-and which is usually not practicable in a field survey). EEG in Ecuador, as in many developing countries is available on a very limited basis only and only $12 \%$ of the 881 definite cases had had a previous EEG. Thus the seizure classification used was not strictly that of the ILAE. ${ }^{17}$ The major effect of the introduction of EEG is to identify a partial onset in apparently generalised tonic clonic seizures. If EEG had been used, a proportion of patients with tonic clonic seizures might have been reclassified as having partial seizures with secondary generalisation.

The estimate that about half of the seizures were partial is higher than in many other epidemiological surveys, ${ }^{411}$ but should still be considered a minimum. The distinction between generalised and partial epilepsy is important from the points of view of investigation and management. Another point is that only two types of seizure were at all 
common; tonic clonic and partial seizures with or without secondarily generalised seizures. Other types of attacks (for example, generalised absence, tonic, and atonic seizures) are uncommon. In teaching rural doctors and other health care personnel in developing countries, it might therefore be of little importance to teach about absence seizure types. It would be better to concentrate on tonic clonic and partial seizures and a simplified approach might lead to a clearer understanding of epilepsy in primary care settings. In countries where most epilepsy is treated in primary care settings, we would advocate a simpler classification scheme, without involvement of EEG. Similarly, for epidemiological work in the future, we would strongly recommend a simplified classification scheme and also the use of standardised instruments. ${ }^{1416}$

The putative aetiologies of epilepsy in this population are based on the medical history only. Only $27 \%$ of cases have an identifiable aetiology and this compares with $39 \%$ in a recent series from the United Kingdom. ${ }^{19}$ Undoubtedly, in a further proportion of cases, an aetiology would have been found after investigation (especially advanced neuroimaging). For instance, in Ecuador, neurocysticercosis is said to be common, and in one hospital series $51 \%$ of patients with secondary epilepsy had the disease as a result of neurocysticercosis. ${ }^{20}$ It is likely that we underestimated neurocysticercosis in this study as the condition is often undetected without investigation.

One of the most interesting aspects is the fact that most of the patients were untreated (86\% of all cases, and $79 \%$ of the active cases) at the time of the survey, or had never received effective treatment $(62 \%$ of all cases). Based on drug supply figures and estimates of prevalence rates and dosage regimens, it had been estimated that only $20 \%$ of the population of patients with active epilepsy in Ecuador would be receiving treatment (a "treatment gap" of $80 \%$ ), and this estimate was shown to be substantially correct, at least for this rural population. ${ }^{10}$ The reasons for this failure to treat are complex, and include such factors as the expectations of the populace regarding treatment, the strength of traditional medicine, the organisation of health care, and the costs of treatment. ${ }^{15}$ It might be argued that the proportion of patients with epilepsy receiving current treatment is an important indicator of the success of a health care system for epilepsy. Further research is needed to identify methods of narrowing the treatment gap and to design a system of health care provision appropriate to the needs and aspirations of the rural population.

It has been suggested that early treatment may in fact influence the course of the condition, and perhaps prevent the development of chronic epilepsy. ${ }^{5-7}$ According to this proposition, treatment may not be simply suppressive, but actually curative in certain situations. If this is the case, there are clearly important implications for health care strate- gies in the control of epilepsy. This proposition is supported by circumstantial evidence only; the finding, for instance, that epilepsy in treated populations tends to go into remission early in its course, and that once in remission, only a small proportion of patients relapse. It is obviously of crucial importance to determine whether this pattern reflects the natural history of epilepsy (with a high rate of spontaneous remission) or is an effect of therapy. Evidence from this study is highly pertinent to this issue, as we are reporting a largely untreated population of patients with epileptic seizures. An important observation can be made: a proportion of cases who never received antiepileptic drugs were free of seizures. Also, a treatment programme was instituted amongst 192 active untreated cases. This was a prospective trial carried out over a 12 month period, and the results and methods of this arm of the study are described in detail elsewhere. ${ }^{21}$ Suffice to say here, that the effectiveness of new treatment with antiepileptic drugs in these previously untreated cases was uniformly excellent and as good as that seen in new cases in developed countries. ${ }^{22-26}$ The results of treatment were as good in patients with a long history of untreated epilepsy as they were in those with a short history. Comparable results have been reported from a similar study carried out in rural Kenya ${ }^{27}$ and from the observation of Watts on the pattern of response to treatment with antiepileptic drugs in patients with a long history of epilepsy in rural Malawi. ${ }^{28} 29$

These findings offer circumstantial evidence against the view that the high remission rates in early epilepsy are due to treatment, and may suggest that this pattern simply reflects the inherent natural history of the condition at least in general cases. Definitive proof of this proposition would require a randomised study of cases treated or not treated at the onset of their epilepsy. We noted a high rate of spontaneous inactivity, seen even in patients never treated. It could be argued that a retrospective study such as this will underreport patients who are free of seizures, and particularly those who had only a small number of seizures in total and those whose seizures were some years ago. It seems likely that such under-reporting will occur, although the numbers of patients developing epilepsy at yearly intervals (the incident cases) in this population were similar over a period of two to 10 years from the survey date, which suggests (assuming incidence rates to be stable over time) that underreporting does not increase over time. ${ }^{18}$ If cases who have been in remission for long periods are under-reported, the tendency towards spontaneous freedom from seizures is even greater than the figures suggest.

We are grateful to the Ministry of Public Health of Ecuador, to the Ecuadorian Society of Neurology, the Ecuadorian League Against Epilepsy, and the National Society for Epilepsy (UK) for their assistance. The study was supported by a grant from the Diseases Control Programme, CibaGeigy, Basel, Switzerland. 
1 Shorvon SD. The temporal aspects of prognosis in epilepsy. $f$ Neurol Neurosurg Psychiatry 1984;47: 1157-65.

2 Annegers JF, Hauser WA, Elveback LR. Remission of seizures and relapse in patients with epilepsy. Epilepsy 1979;20:729-37.

3 Goodridge DMG, Shorvon SD. Epilepsy in a population of 6000. BMF 1983;287:641-7.

4 Sander JWAS, Shorvon SD. Incidence and prevalence studies in epilepsy and their methodological problems: a review. F Neurol Neurosurg Psychiatry 1987;50:829-39.

5 Shorvon SD. Do anticonvulsants influence the natural history of epilepsy? In: Warlow C, Garfield J, eds. More tory of epilepsy? In: Warlow C, Garfield J, eds. More dilemmas in the management of the neurological
Edinburgh: Churchill Livingstone, 1987; 8-13.

6 Reynolds $\mathrm{EH}$. Early treatment and prognosis of epilepsy. Epilepsia 1987;28:97-106.

7 Elwes RDC, Reynolds EH. The early prognosis of epilepsy. In: Dam M, Gram L, eds. Comprehensive epileptology. New York: Raven Press 1990; 715-27.

8 Schoemberg BS. Clinical neuroepidemiology in developing countries: neurology with few neurologists. Neuroepidemiology 1982;1:137-42.

9 Osuntokun BO, Adeuja AOG, Nottidge VA, et al. Prevalence of the epilepsies in Nigerian Africans: A community-based study. Epilepsia 1987;28:272-9.

10 Shorvon SD, Farmer PJ. Epilepsy in developing countries: a review of epidemiological, sociocultural and treatment a review of epidemiological, sociocult

$11 \mathrm{Li} \mathrm{SC}$, Schoemberg BS, Bolis CL, et al. Epidemiology of epilepsy in urban regions of the People's Republic of China. Epilepsia 1985;26:391-4.

12 Bharucha NE, Bharucha E, Bharucha AE, et al. Prevalence of epilepsy in the Parsi community of Bombay. Epilepsia 1988;29:111-5.

13 Koul R, Razdan S, Motta A. Prevalence and pattern of epilepsy (Lath/Mirgi/Laran) in Rural Kashmir, India. Epilepsia 1988;29:116-22.

14 Placencia M, Suarez J, Crespo F, Sander JWAS, Shorvon SD, Ellison RH. A large scale study of epilepsy in Ecuador: methodological aspects. Neuroepidemiology 1992;11:74-84.

15 Farmer P, Placencia M, Jumbo L, Sander JWAS, Shorvon SD. Help-seeking strategies for epilepsy by previously untreated patients in Northern Ecuador. Epilepsia Res untreated patients

16 Placencia M, Sander JWAS, Shorvon SD, Ellison RH.
Validation of a screening questionnaire for the detection of epilepsy in epidemiological studies. Brain 1992;115 783-94.

17 Commission on classification and terminology of the International League of epilepsy. Proposal for classification of epilepsy and epileptic syndromes. Epilepsia 1985;26:268-78.

18 Placencia M, Shorvon SD, Paredes V, et al. Epileptic seizures in an Andean region of Ecuador: prevalence and incidence and regional variation. Brain 1992;115: and incide.

19 Sander JWAS, Hart YM, Johnson AL, Shorvon SD. The National General Practice Study of Epilepsy: newly diagnosed seizures in a general population. Lancet 1990,336:1267-71

20 Del Bruto OH, Santibanez R, Noboa CA, Aguirre R, Diaz E, Alarcon TA. Epilepsy due to neurocysticercosis: analysis of 203 patients. Neurology 1992;42:389-92.

21 Placencia M, Sander JWAS, Alarcon F, Cascante S, Shorvon SD. A prospective antiepileptic treatment programme in a largely previously untreated population. Epilepsia Res 1993;14:237-44.

22 Turnbull DM, Rawlins MD, Weightman D, Chadwick $\mathrm{DW}$. A comparison of phenytoin and valproate in previously untreated adult epileptic patients. $f$ Neurol Neurosurg Psychiatry 1982;55-9.

23 Shorvon SD, Reynolds EH. Early prognosis of epilepsy. BMF 1982;285:1699-701.

24 Elwes RDC, Johnson AL, Shorvon SD, Reynolds EH. The prognosis of seizure control in newly diagnosed epilepsy. N Engl f Med 1984;311:944

25 Elwes RDC, Chesterman P, Reynolds EH. Prognosis after a first untreated toni-clonic seizure. Lancet 1985;ii 752-3.

26 Callaghan N, Kenny R, O'Neill B, et al. A prospective study between carbamazepine, phenytoin and sodium valproate as monotherapy in previously untreated and recently diagnosed patients with epilepsy. $f$ Neurol Neurosurg Psychiatry 1985;48:639-44.

27 Feksi AT, Kaamugisha J, Gatiti S, Sander JWAS, Shorvon SD. Comprehensive primary health care antiepileptic drug treatment in rural and semi-urban Kenya. Lancet 1991,337:406-7.

28 Watts AE. A model for managing epilepsy in a rural community in Africa. $B M \mathcal{T}$ 1989;298:805-7.

29 Watts AE. The natural history of untreated epilepsy in a rural community in Africa. Epilepsia 1992;33:464-8. 